\title{
Changes in synaptic plasticity are associated with electroconvulsive shock-induced learning and memory impairment in rats with depression-like behavior
}

This article was published in the following Dove Press journal:

Neuropsychiatric Disease and Treatment

\section{Qibin Chen' \\ Li Ren' \\ Su Min' \\ Xuechao $\mathrm{Hao}^{2}$ \\ Hengsheng Chen ${ }^{3}$ \\ Jie Deng'}

'Department of Anesthesiology, The First Affiliated Hospital of Chongqing Medical University, Chongqing, ${ }^{2}$ Department of Anesthesiology, West China Hospital of Sichuan University, Chengdu, Sichuan, ${ }^{3}$ Ministry of Education Key Laboratory of Child Development and Disorders, China International Science and Technology Cooperation Base of Child Development and Critical Disorders, Chongqing Key Laboratory of Pediatrics, Chongqing, People's Republic of China
Correspondence: Su Min Department of Anesthesiology, The First Affiliated Hospital of Chongqing Medical University, No I Youyi Road, Yuzhong District, Chongqing 4000I6, People's Republic of China Tel/fax +86 23 890I 1068 Emailms89011068@I63.com
Background: Accompanied with the effective antidepressant effect, electroconvulsive shock (ECS) can induce cognitive impairment, but the mechanism is unclear. Synaptic plasticity is the fundamental mechanism of learning and memory. This study aimed to investigate the effect of ECS on synaptic plasticity changes in rats with depression-like behavior.

Methods: Chronic unpredictable mild stress procedure was conducted to establish a model of depression-like behavior. Rats were randomly divided into the following three groups: control group with healthy rats (group C), rats with depression-like behavior (group D), and rats with depression-like behavior undergoing ECS (group DE). Depression-like behavior and spatial learning and memory function were assessed by sucrose preference test and Morris water test, respectively. Synaptic plasticity changes in long-term potentiation (LTP), long-term depression (LTD), depotentiation, and post-tetanic potentiation (PTP) were tested by electrophysiological experiment.

Results: ECS could exert antidepressant effect and also induced spatial learning and memory impairment in rats with depression-like behavior. And, data on electrophysiological experiment showed that ECS induced lower magnitude of LTP, higher magnitude of LTD, higher magnitude of depotentiation, and lower magnitude of PTP.

Conclusion: ECS-induced learning and memory impairment may be attributed to postsynaptic mechanism of LTP impairment, LTD and depotentiation enhancement, and presynaptic mechanism of PTP impairment.

Keywords: electroconvulsive therapy, learning, memory, synaptic plasticity, electrophysiology

\section{Introduction}

Depression has been ranked as the third disease burden in the world, and $>1$ million people commit suicide each year due to depression. ${ }^{1,2}$ Although antidepressants are the first-line treatment for depression, approximately one-third of patients, especially with major or refractory depression, are not responsive to the medication. ${ }^{3}$ Electroconvulsive therapy (ECT) is conducted as the most effective treatment for those with major or drug-resistant depression. Nevertheless, accompanied with its excellent treatment effect, ECT can induce learning and memory impairment, which is a major limitation in the clinical use of ECT. ${ }^{4}$ Unfortunately, the underlying mechanism of learning and memory impairment induced by ECT is still poorly understood.

Persistent neural modifications are widely regarded as the cellular basis for learning and memory. ${ }^{5}$ Synaptic plasticity is the fundamental mechanism of neural modifications, 
and synaptic plasticity supporting learning and memory process involves persistent changes in synaptic efficacy such as long-term potentiation (LTP) and long-term depression (LTD). ${ }^{6,7}$ Although LTP is remarkable for its stability, accumulative evidence revealed that LTP could be reversed if applied shortly after LTP induction and this form of synaptic plasticity was named as depotentiation. ${ }^{8}$ Depotentiation has been reported in hippocampus $s^{9,10}$ and other brain regions closely related to learning and memory, such as prefrontal cortex $^{11}$ and amygdala. ${ }^{12}$ Thus, it is universally accepted that LTP, LTD, and depotentiation conduct as opposing mechanisms that maintain a dynamic range of synaptic efficacy and keep synaptic homeostasis. ${ }^{13,14}$ Our previous study found that electroconvulsive shock (ECS, an analog of ECT to animals) could induce LTP impairment. ${ }^{15}$ However, whether LTD or depotentiation contributes to ECT-induced learning and memory impairment is still unknown.

Virtually, all synapses are mediated by short-lived and long-lasting process. Thus, besides the long-term modifications of synaptic plasticity, changes in short-term synaptic plasticity also have an important role in learning and memory. Post-tetanic potentiation (PTP) is a widespread form of short-term synaptic plasticity, which suggests that synaptic efficacy is enhanced for tens of seconds to minutes after high-frequency stimulation (HFS) with a train of hundreds of pulses. ${ }^{16}$ Furthermore, it has been proved that presynaptic transmitters' release is reflected by PTP.${ }^{17}$ Our previous study found that ECS-induced learning and memory impairment was attributed to imbalance of hippocampal Glu/GABA. ${ }^{18}$ However, it is still unclear whether PTP is involved in the learning and memory impairment induced by ECT. Besides, how transmitter release influences the learning and memory function in the model of ECS necessitates further study.

In this study, using behavioral test and electrophysiological experiments, we investigate the effects of ECS on learning and memory as well as the potential roles of LTP, LTD, depotentiation, and PTP in this process.

\section{Methods}

\section{Animals}

Healthy adult male Sprague Dawley rats (weight 200-250 g, aged 2-3 months) were obtained from the Laboratory Animal Center of Chongqing Medical University. All rats were housed at standard condition (temperature at $22^{\circ} \mathrm{C} \pm 2{ }^{\circ} \mathrm{C}$, humidity at $62 \% \pm 3 \%$, and $12 / 12 \mathrm{~h}$ light-dark cycle) in the animal room. The rats were kept for 7 days acclimation period before experiment. All experiment protocols were approved by the Ethical Committee of the First Affiliated
Hospital of Chongqing Medical University (No 2017-004) and conducted according to National Institutes of Health's Guild for the Care and Use of Laboratory Animals.

\section{Rats' model of depression-like behavior}

As previously described, chronic unpredictable mild stress (CUMS) procedure was conducted to establish a model of depression-like behavior in rats. ${ }^{19}$ All rats except for those in group $\mathrm{C}$ were housed in individual cages and exposed the following stress: food deprivation for $24 \mathrm{~h}$, water deprivation for $24 \mathrm{~h}$, continuous lighting for $24 \mathrm{~h}$, tailing pinching for $1 \mathrm{~min}, 5 \mathrm{~min}$ swimming in the cold water of $4^{\circ} \mathrm{C}, 5 \mathrm{~min}$ swimming in the hot water of $45^{\circ} \mathrm{C}$, shaking for $20 \mathrm{~min}$, damp sawdust for $24 \mathrm{~h}$, and cages tilting to $45^{\circ}$ for $24 \mathrm{~h}$. To avoid habituation and provide an unpredictable feature to the stressors, all the stressors were randomly scheduled and repeated within 4 weeks.

\section{Experiment groups and treatment}

Rats were randomly divided into the following three groups: group $\mathrm{C}$ includes the healthy rats without any treatment, group D includes the depression-like behavior rats and treated with sham ECS, and group DE includes the depression-like behavior rats and treated with ECS. A total of 32 rats were included for each group. ECS was conducted via bilateral ear clip electrodes with a Niviqure ECS system (Nivique Meditech, Bangalore, India) on the basis of following parameters: bidirectional square wave pulses, $0.8 \mathrm{~A}$ in amplitude, $1.5 \mathrm{~ms}$ in width, $125 \mathrm{~Hz}$ in frequency, and $0.8 \mathrm{~s}$ in duration, and $120 \mathrm{mC}$ charge. Sham ECS was conducted as the same process with ECS but without currents. The ECS or sham ECS was conducted once daily for 7 days.

\section{Sucrose preference test}

Sucrose preference test is widely accepted to test anhedonia, which is one of the core symptoms of depression to evaluate the depression-like behavior in rats. The test was conducted as previously described. ${ }^{20}$ The test was completed within $72 \mathrm{~h}$. In the first $24 \mathrm{~h}$, rats were kept with two bottles of $1 \%$ sucrose solution to adapt sucrose consumption. In the second $24 \mathrm{~h}$, rats were kept with one bottle of $1 \%$ sucrose solution and one bottle of sterile water. In the third $24 \mathrm{~h}$, after water and food deprivation for $23 \mathrm{~h}$, all rats were kept with two identical and preweighed bottles for $1 \mathrm{~h}$. One bottle was filled with $1 \%$ sucrose solution, and another bottle was filled with sterile water. After $30 \mathrm{~min}$ free drinking, the position of two bottles was exchanged to prevent the position preference. The sucrose preference 
percentage (SPP) was calculated as the following formula: SPP $(\%)=$ sucrose consumption $(\mathrm{g}) /($ water consumption $[\mathrm{g}]+$ sucrose consumption $[\mathrm{g}]) \times 100 \%$.

\section{Morris water maze}

It is well received that hippocampus-dependent spatial learning and memory function of rats can be assessed by Morris water maze. ${ }^{21} \mathrm{~A}$ circle pool $(150 \mathrm{~cm}$ in diameter and $50 \mathrm{~cm}$ in height) was filled with water colored by black ink, and a submerge platform ( $11 \mathrm{~cm}$ in diameter) was provided below the surface of the water with $\sim 1-2 \mathrm{~cm}$. The pool was divided into four quadrants, and each rat was placed into the water randomly at one of the four quadrants to find the hidden platform within $60 \mathrm{~s}$. If the rats did not find the platform within $60 \mathrm{~s}$, they were guided to the platform with a stick and stayed on the platform for $15 \mathrm{~s}$. Each rat was placed into the water gently from four different quadrants once a day for 5 consecutive days. The time to find the platform for each quadrant was defined as escape latency, and average time from four quadrants of the consecutive 5 day was analyzed. On the sixth day, the platform was removed and rats were placed into water to swim with a limitation of $60 \mathrm{~s}$. Space exploration time, defined as swimming time in the platform quadrant of each rat, was recorded. Escape latency, space exploration time, and swimming speed based on the swimming track recorded by a video track system program (Zhenghua Instruments, Anhui, China) were analyzed. A plain experimental timeline for all behavior tests is shown in Figure 1A.

\section{Hippocampal slice preparation}

Rats were anesthetized by $2 \%$ pentobarbital sodium, and the brain was removed gently. Hippocampal slices ( $400 \mu \mathrm{m}$ thick) were cut by a vibratome (NVSLM1; WPI, Sarasota, FL, USA) in $0^{\circ} \mathrm{C}-4^{\circ} \mathrm{C}$ cutting solution oxygenated with the mix gas of
95\% $\mathrm{O}_{2}$ and $5 \% \mathrm{CO}_{2}$. Cutting solution was configured as follows (in millimolar): $3 \mathrm{KCl}, 1.25 \mathrm{NaH}_{2} \mathrm{PO}_{4}, 26 \mathrm{NaHCO}_{3}$, 0.4 vitamin C, 2 sodium pyruvate, 2 sodium lactate, 10 glucose, 220 sucrose, $1 \mathrm{MgCl}_{2}, 1 \mathrm{CaCl}_{2}, 1 \mathrm{MgSO}_{4}$ (pH 7.3-7.4; osmotic pressure $300-310 \mathrm{mOsmol} / \mathrm{L}$ ). Slices were incubated in a chamber filled with oxygenated recording solution at $34^{\circ} \mathrm{C}$ for $60 \mathrm{~min}$ and then incubated in identical solution at $24^{\circ} \mathrm{C}$ for at least $60 \mathrm{~min}$ before recording. Recording solution was comprised as follows (in millimolar): $124 \mathrm{NaCl}, 3 \mathrm{KCl}, 1.25$ $\mathrm{NaH}_{2} \mathrm{PO}_{4}, 26 \mathrm{NaHCO}_{3}, 0.4$ vitamin $\mathrm{C}, 2$ sodium pyruvate, 2 sodium lactate, 10 glucose, $1 \mathrm{CaCl}_{2}, 1 \mathrm{MgSO}_{4}$ (pH 7.3-7.4; osmotic pressure 300-310 mOsmol/L).

\section{Electrophysiological experiment}

A single hippocampal slice was placed in the recording chamber and was continuously perfused with oxygenated recording solution. A bipolar stimulating electrode was placed in the stratum radiatum of $\mathrm{CA} 3$ region, and a glass micropipette (resistance 2-3 M 2 ) filled with filtrated recording solution was placed in the stratum radiatum of CA1 region to record the field excitatory postsynaptic potentials (fEPSPs). Stimulation intensity to evoke baseline fEPSPs was set as $\sim 50 \%$ of the intensity, which elicited the maximal response. As previously described, after 30 min stable baseline fEPSPs' recording, LTP was induced by an HFS with the parameter of 400 pulses at $100 \mathrm{~Hz} .{ }^{22}$ To assess the magnitude of LTP, the mean value for the slope of fEPSPs recorded at 20-40 min after stimulation was calculated and expressed as a percentage of the mean value of the initial baseline slope of fEPSPs. Depotentiation was induced as previously described. ${ }^{23}$ After successful LTP induction, the same slice received a lowfrequency stimulation (LFS) (900 pulses at $1 \mathrm{~Hz}$ ) to induce depotentiation $($ depotentiation $=$ [percentage of potentiation after LFS -100$] \times 100 /[$ percentage of potentiation before LFS -100]). For LTD induction, after stable baseline fEPSPs
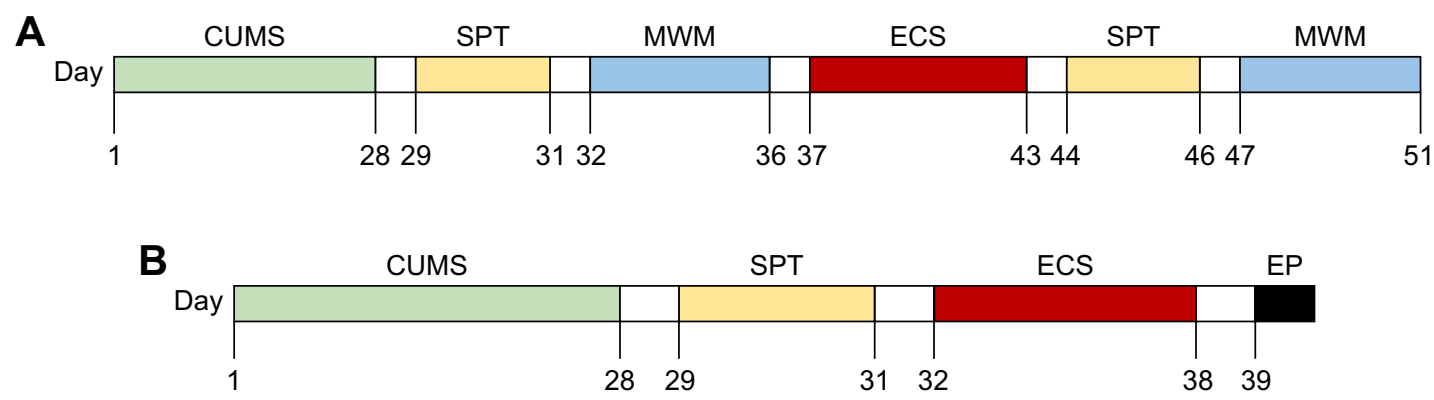

Figure I Time overview of the study.

Notes: (A) The time schedule for behavior test. (B) The time schedule for electrophysiological experiment.

Abbreviations: CUMS, chronic unpredictable mild stress; ECS, electroconvulsive shock; EP, electrophysiological experiment; MWM, Morris water maze; SPT, sucrose preference test. 
recording for $30 \mathrm{~min}$, LTD was induced by a LFS (900 pulses at $1 \mathrm{~Hz}) \cdot{ }^{23}$ The calculation of LTD was similar as that of LTP. All the poststimulation fEPSPs of LTP, depotentiation and LTD were recorded for $60 \mathrm{~min}$. PTP was induced as previously described with minor modification. ${ }^{24}$ After $30 \mathrm{~s}$ stable baseline fEPSPs recording, PTP was induced by an HFS (400 pulses at $400 \mathrm{~Hz}$ ) and the poststimulation fEPSPs were recorded for $100 \mathrm{~s}$. The mean value for the slope of fEPSPs recorded at $5 \mathrm{~s}$ after stimulation was calculated, and PTP was expressed as a percentage of the mean value of the initial baseline slope of fEPSPs. All electrophysiological data were recorded and analyzed by Axon Instruments system (MultiClamp 700B amplifier, Digidata 1200 transverter, pCLAMP 9.2 software; Molecular Devices LLC, Sunnyvale, CA, USA). A scheduled timeline for electrophysiological experiment is shown in Figure 1B.

\section{Statistical analysis}

Statistical analysis was performed with SPSS (Version 17.0; SPSS Inc., Chicago, IL, USA). All data were expressed as mean \pm SD. Statistical significance was determined by repeated measures analysis of variance (data of escape latency from Morris water maze). The other data were analyzed by one-way analysis of variance, followed by Bonferroni correction to compare differences between the groups. $P<0.05$ was considered statistically significant.

\section{Results}

\section{Along with the antidepressant effect, ECT- induced learning and memory impairment}

First, we want to clear the effect of ECS on behavior changes. Eight rats in each group were used to behavior test. Depression-like behavior was tested by sucrose preference test. As shown in Figure 2A, group D and group DE shown lower SPP than group C after CUMS procedure $(F=42.102$, post hoc test, $P<0.001$ and $<0.001$, respectively). After ECS treatment, SPP of group DE was higher than that of group D ( $F=36.695$, post hoc test, $P<0.001$ ); however, it showed no difference compared with group $\mathrm{C}$ (post hoc test, $P=0.810$ ). Spatial learning and memory function was tested by Morris water maze. There was no difference in the comparison of swimming speed for each group (data were not shown). Escape latency decreased gradually during the 5 days training for the three groups. However, rats in group DE spent more

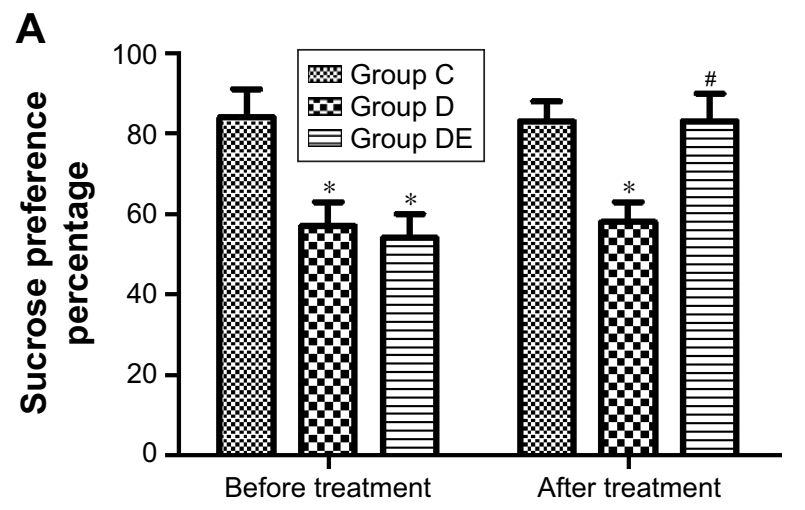

B
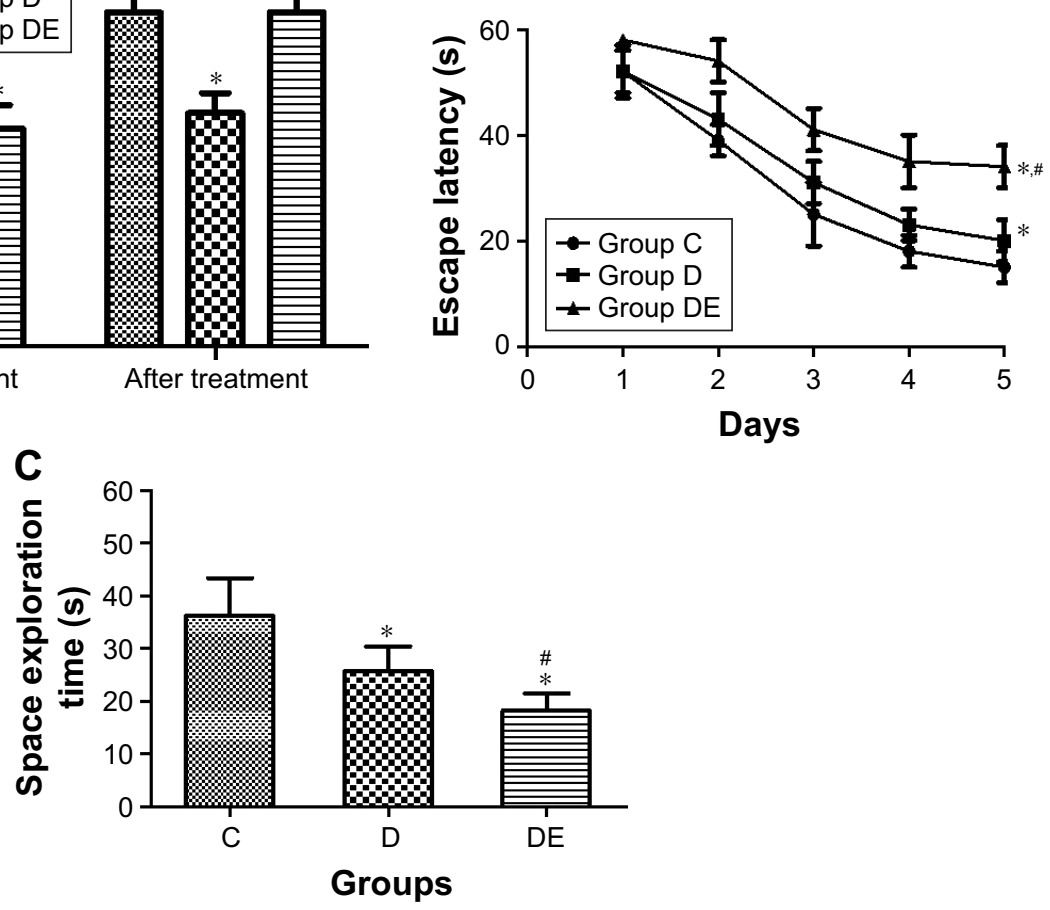

Notes: Sucrose preference percentage is the test index of sucrose preference test, and lower sucrose preference percentage indicated more significant depression-like behavior. Escape latency and space exploration time are the test index of Morris water maze, and longer escape latency and shorter space exploration time indicated worse learning and memory performance. Group C, control group with healthy rats; Group D, rats with depression-like behavior; Group DE, rats with depression-like behavior undergoing ECS. ${ }^{*} P<0.05$ compared with group $C$; ${ }^{\#} P<0.05$ compared with group $D$.

Abbreviation: ECS, electroconvulsive shock. 
time to find the platform than other two groups $(F=111.803$, post hoc test, $P<0.001$ and $<0.001$, respectively). Furthermore, escape latency of group $\mathrm{D}$ was longer than that of group C (post hoc test, $P=0.001$ ) (Figure 2B). Data of space exploration time suggested that group DE spent least time to swim in the platform quadrant $(F=17.394$, post hoc test, $P<0.001$ and 0.027 , respectively). What is more, group D showed shorter space exploration time than group $\mathrm{C}$ (post hoc test, $P=0.004$ ) (Figure $2 \mathrm{C}$ ).

\section{ECS-induced LTP impairment and depotentiation enhancement}

To illuminate the underlying electrophysiological mechanisms of learning and memory impairment induced by ECS, LTP and depotentiation were assessed. Morris water maze itself was a process of learning and memory. To prevent the potential effect of Morris water maze on subsequent electrophysiological assessment, another eight rats in each group were included. As shown in Figure 3, baseline fEPSP of group DE (fEPSP slope, $0.206 \pm 0.196, F=85.683$, post hoc test, $P<0.001$ ) was higher than that of group $C$ and group $\mathrm{D}$. No difference was found in the comparison of baseline fEPSP between group C (fEPSP slope, 0.118 \pm 0.014 ) and group D (fEPSP slope, $0.100 \pm 0.009$, post hoc test, $P=0.053$ ), although the baseline fEPSP was lower in group D. For LTP test, group DE (normalized fEPSP slope, 129\% $\pm 4 \%$ ) showed lower magnitude of LTP than group D (normalized fEPSP slope, $166 \% \pm 5 \%, F=119.934$, post hoc test, $P<0.001)$ and group C (normalized fEPSP slope, $184 \% \pm 8 \%$, post hoc test, $P<0.001)$. Compared with group $\mathrm{C}$, the magnitude of LTP was lower in group D (post hoc test, $P<0.001$ ). For depotentiation test, $\sim 50 \%$ LTP was reversed by LFS in group C (normalized fEPSP slope, 42\% $\%$ 7\%). Compared with group $\mathrm{C}$, depotentiation was enhanced in group $\mathrm{D}$ (normalized fEPSP slope, $69 \% \pm 5 \%, F=83.438$, post hoc test, $P<0.001$ ) and group DE (normalized fEPSP slope, $89 \% \pm 5 \%$, post hoc test, $P<0.001)$. Furthermore, group DE exhibited stronger depotentiation than group D (post hoc test, $P<0.001)$.

\section{ECS-induced LTD enhancement}

LTD is another important form of synaptic plasticity and also plays a central role in learning and memory. In our experiment, group DE (normalized fEPSP slope, 58\% 0 \% ) exhibited higher magnitude of LTD compared with group D (normalized fEPSP slope, $68 \% \pm 7 \%, F=12.438$, post hoc test, $P=0.025$ ) and group C (normalized fEPSP slope, $77 \% \pm 7 \%$, post hoc test, $P<0.001)$. What is more, compared with group $\mathrm{D}$, the magnitude of LTD was lower in group $\mathrm{C}$ (post hoc test, $P=0.025$ ) (Figure 4).

\section{ECS-induced PTP impairment}

LTP, LTD, and depotentiation are forms of postsynaptic plasticity; however, we wanted to clear whether presynaptic plasticity, such as PTP, was related to the learning and memory impairment induced by ECS. As shown in Figure 5, the magnitude of PTP of group DE (normalized fEPSP slope, $121 \% \pm 9 \%$ ) was lower than that of group $C$ (normalized fEPSP slope, $150 \% \pm 7 \%, F=23.927$, post hoc test, $P<0.001$ ) and group D (normalized fEPSP slope, $140 \% \pm 5 \%$, post hoc test, $P<0.001)$. Compared with group $\mathrm{C}$, the magnitude of PTP was lower in group D (post hoc test, $P=0.034$ ).

\section{Discussion}

The present study confirmed that along with the antidepressant effect, ECS induced learning and memory impairment in the rats' model of depression-like behavior. ECS induced synaptic plasticity changes including LTP impairment, LTD and depotentiation enhancement, and PTP impairment in rats with depression-like behavior, leading to learning and memory impairment.

It has been proved that measures, such as learned helpless, ${ }^{25}$ olfactory bulbectomy, ${ }^{26}$ maternal separation, ${ }^{27}$ and CUMS, ${ }^{28}$ can induce depression-like behavior of rats. In our study, depression-like behavior model was constructed by CUMS because this model can better simulate the process of the depression induced by environmental stress, which is one of the most common pathogeneses of depression. ${ }^{29}$ Anhedonia is one the central symptoms of depression and can be assessed by SPP for rats. In line with other study, ${ }^{30}$ our results revealed that SPP decreased significantly after CUMS procedure and ECS increased the SPP in depression-like behavior model of rats. Additionally, we found that ECS could increase the baseline fEPSP, which was defined as "LTP-like" changes and considered as the underlying mechanism of antidepressant of ECS. ${ }^{31,32}$ The trend of baseline fEPSP was consistent with the results of SPP, indicating successful model construction and antidepressant effect of ECS.

In Morris water maze test, we found rats with depressionlike behavior undergoing ECS exhibited learning and memory impairment. LTP and LTD are two common electrophysiological forms of synaptic plasticity. In our experiment, we found that ECS induced LTP impairment. In the test of LTD, to our surprise, the magnitude of LTD in group DE was larger than that in group D, indicating that ECS induced LTD 
A
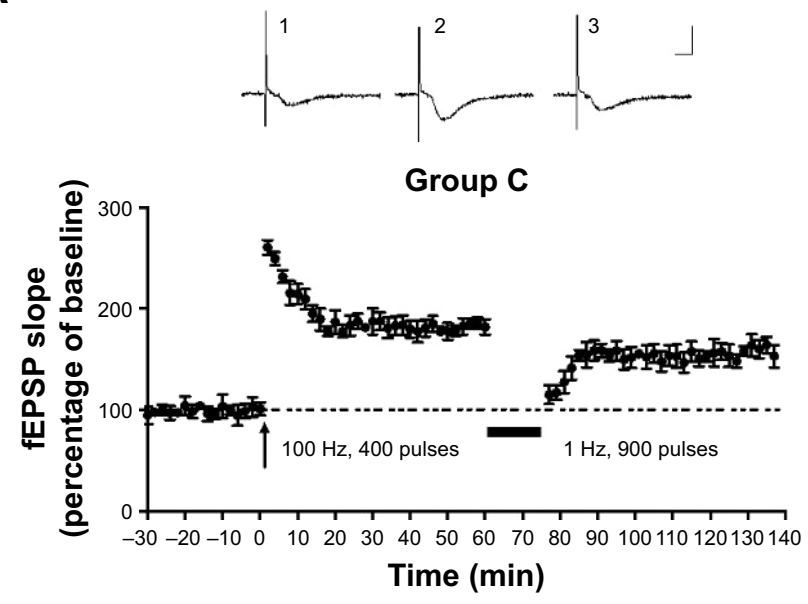

C

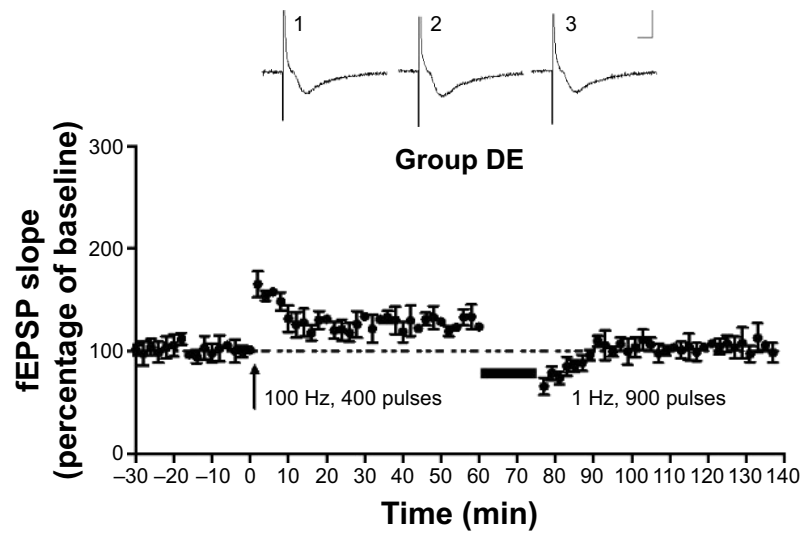

E

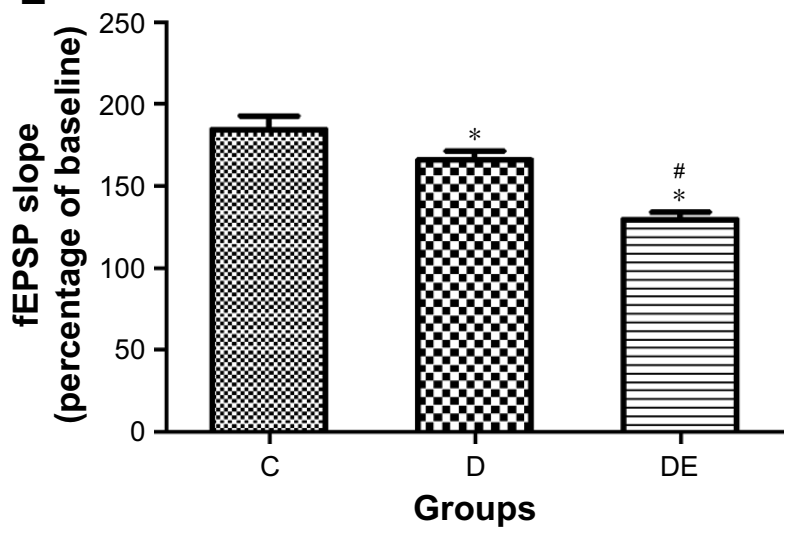

B

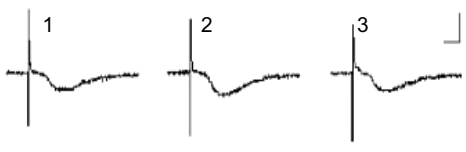

Group D

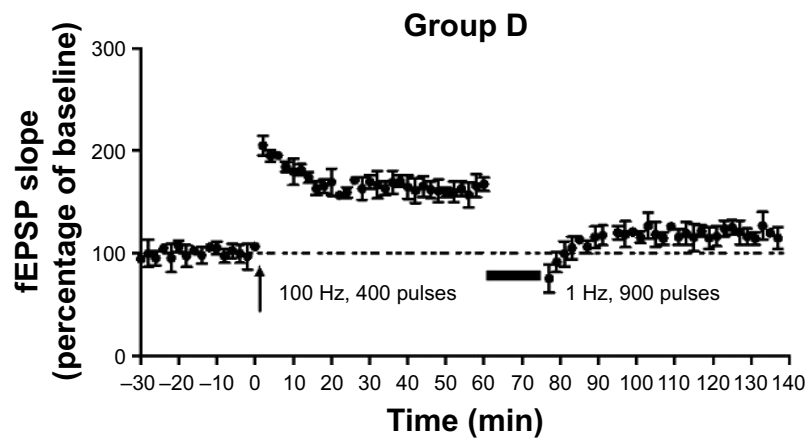

D

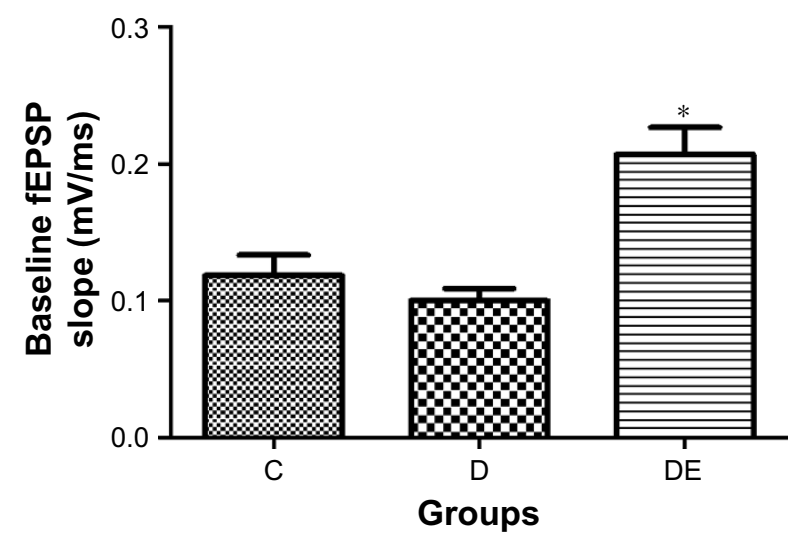

F

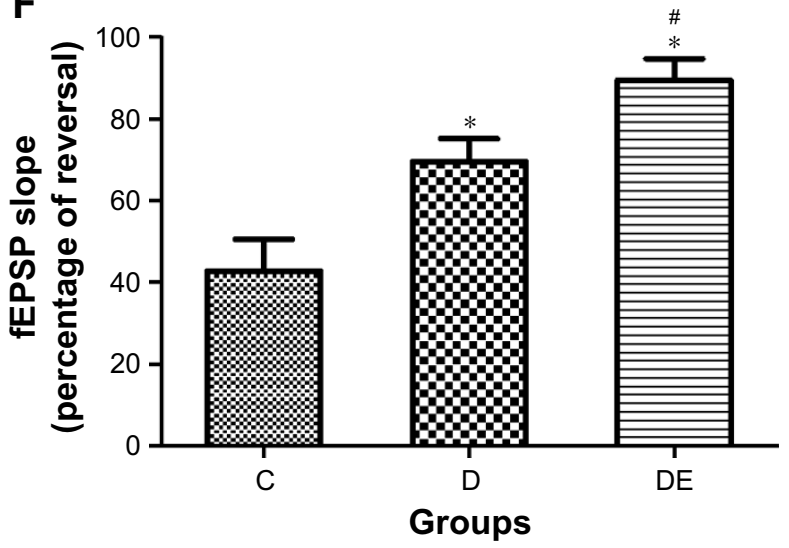

Figure 3 Effects of ECS on LTP and depotentiation.

Notes: Baseline fEPSPs were recorded for $30 \mathrm{~min}$, and poststimulation fEPSPs of LTP and depotentiation were recorded for 60 min. (A-C) LTP and depotentiation were recorded in each group. Original traces of fEPSP were recorded, and baseline trace (trace I), post-HFS trace (trace 2), and post-LFS trace (trace 3) were exhibited. Scale bar was set as $5 \mathrm{~ms}$ for the horizontal line and I $\mathrm{mV}$ for the vertical line. Statistical analysis of baseline fEPSP (D), LTP (E), and depotentiation (F) for different groups. Group C, control group with healthy rats; Group D, rats with depression-like behavior; Group DE, rats with depression-like behavior undergoing ECS. $* P<0.05$ compared with group C; ${ }^{P}<0.05$ compared with group $D$.

Abbreviations: ECS, electroconvulsive shock; fEPSPs, field excitatory postsynaptic potentials; LTP, long-term potentiation; LFS, low-frequency stimulation; HFS, highfrequency stimulation.

enhancement. Briefly, ECS induced LTP inhibition and LTD enhancement. Accumulative evidence suggested that LTD or LTP induction could be influenced by the history of synaptic activity. This form of regulation of synaptic plasticity has been named as "plasticity of plasticity" or "metaplasticity", which reveals that prime synaptic activation will suppress subsequent LTP and facilitate LTD via LTD/LTP threshold sliding to right. ${ }^{33}$ In the present study, ECS could induce 
A

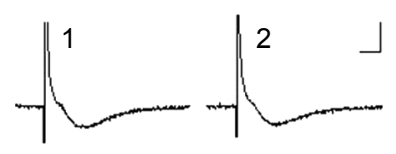

월

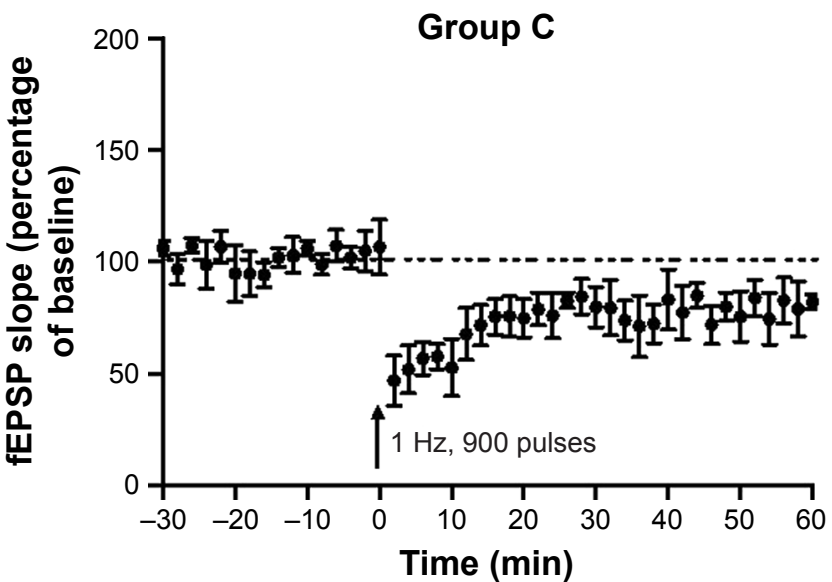

C

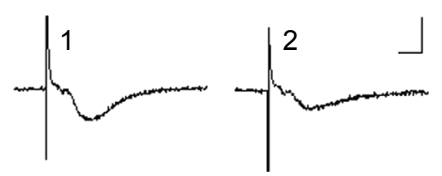

Group DE

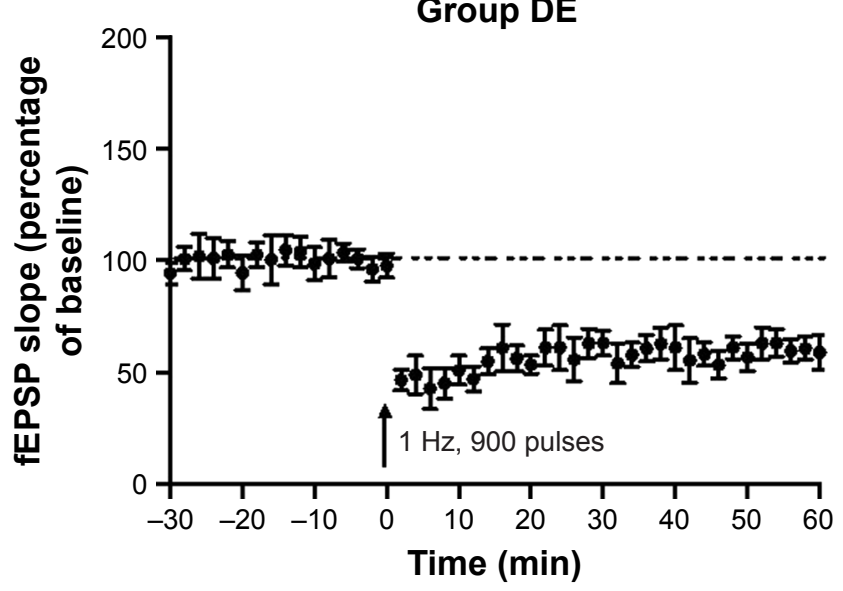

B
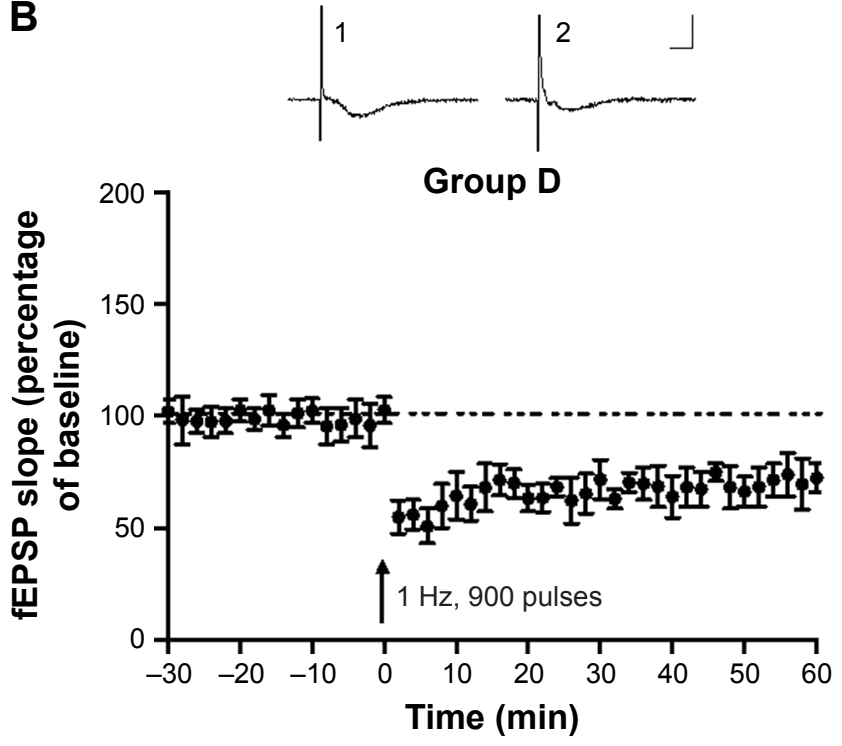

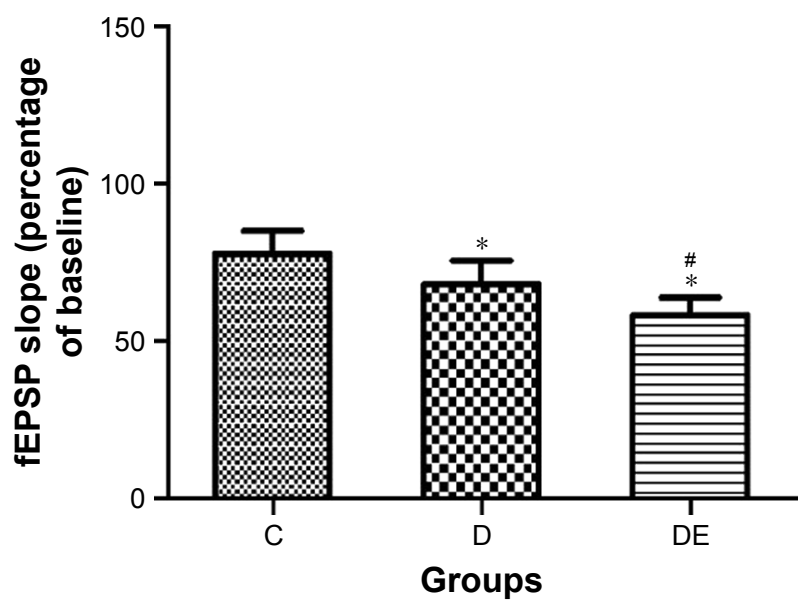

Figure 4 Effects of ECS on LTD

Notes: Baseline fEPSPs were recorded for $30 \mathrm{~min}$, and poststimulation fEPSPs were recorded for $60 \mathrm{~min}$. (A-C) LTD was recorded in each group. Original traces of fEPSP were recorded, and baseline trace (trace I) and poststimulation trace (trace 2) were exhibited. Scale bar was set as $5 \mathrm{~ms}$ for the horizontal line and I $\mathrm{mV}$ for the vertical line. (D) Statistical analysis of LTP for different groups. Group C, control group with healthy rats; Group D, rats with depression-like behavior; Group DE, rats with depressionlike behavior undergoing ECS. *P<0.05 compared with group $C$; ${ }^{P}<0.05$ compared with group.

Abbreviations: ECS, electroconvulsive shock; fEPSPs, field excitatory postsynaptic potentials; LTD, long-term depression; LTP, long-term potentiation.

baseline fEPSP increase, indicating synaptic activation by ECS. ${ }^{31}$ And, our previous study also found that ECS could upregulate the expression of pT305-CaMKII, which is closely related to LTD/LTP threshold regulation. ${ }^{34}$ All these results strongly suggested that ECS-induced learning and memory impairment could not be simply explained by synaptic plasticity. To the best of our knowledge, alternation of regulatory capacity of synaptic plasticity or metaplasticity contributes to learning and memory impairment induced by ECS indeed..$^{35}$

In our study, it was confirmed that rats with depressionlike behavior also exhibited learning and memory impairment coincided with LTP impairment and LTD enhancement. Thus, it is eligible to receive that learning and memory impairment induced by depression and ECS involved in the regulation of metaplasticity. In addition, our results revealed that more serious LTP impairment and LTD enhancement were found in group DE. In consideration of previous finding that stress could induce LTP impairment and LTD enhancement, ${ }^{35,39} \mathrm{a}$ reasonable explanation is that stress of different intensities induces different magnitudes of LTP impairment and LTD enhancement. Acute strong enough stress, such as electronic stimulation with ECS, will induce serious LTP impairment and LTD enhancement; however, chronic or mild stimulation, 
A
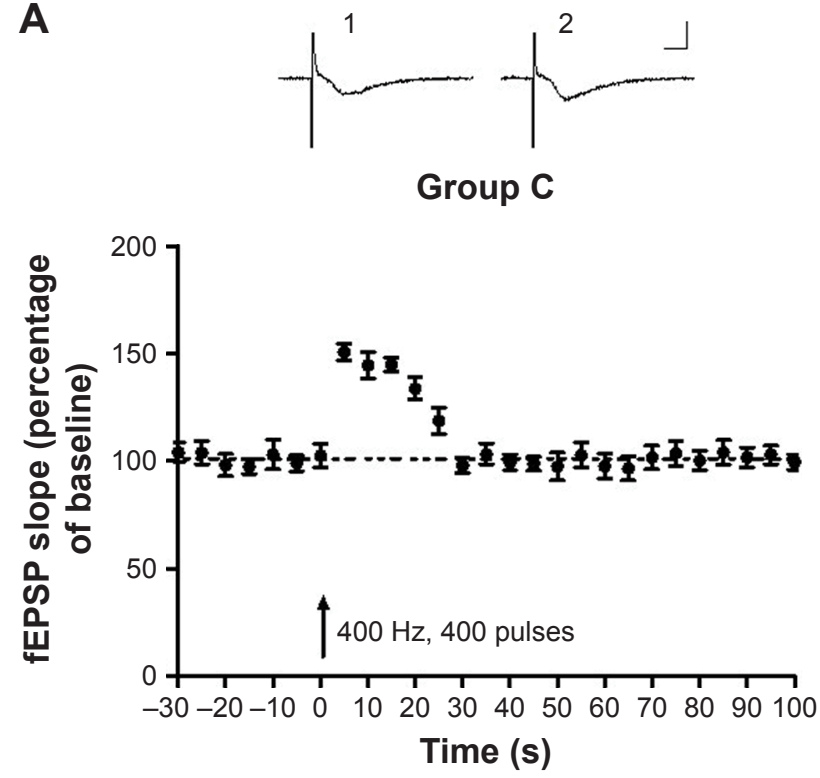

C

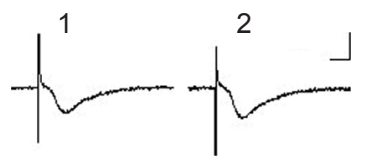

Group DE

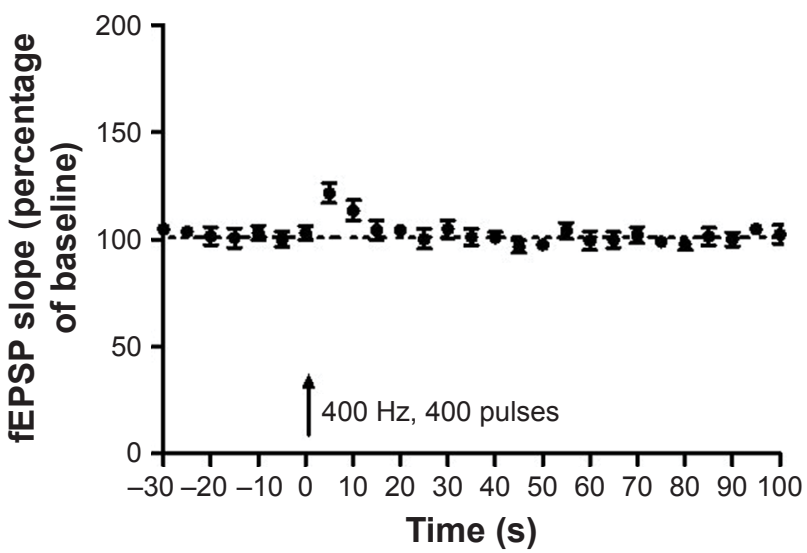

B
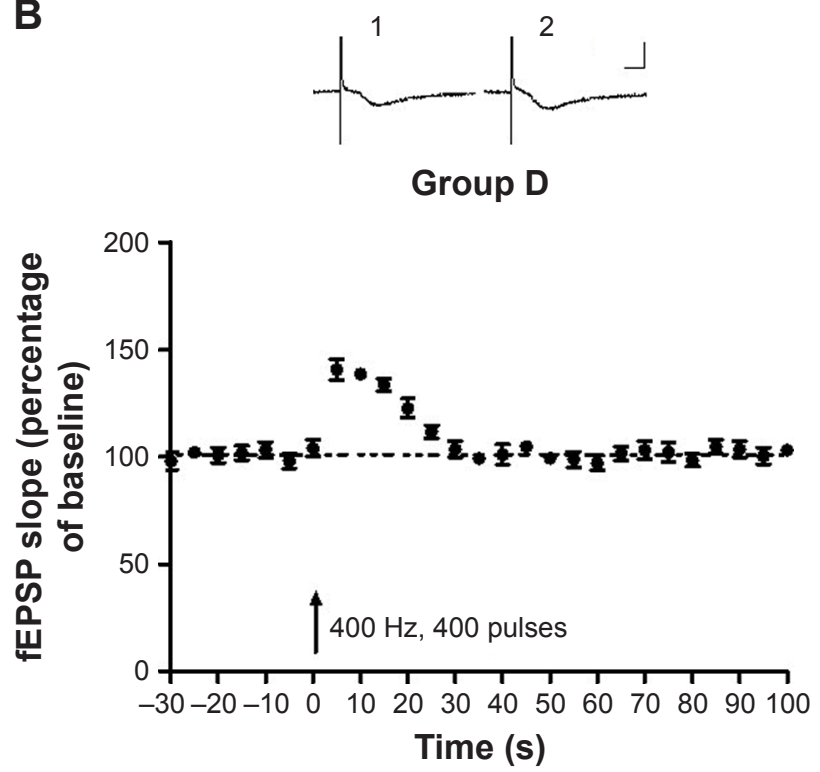

D

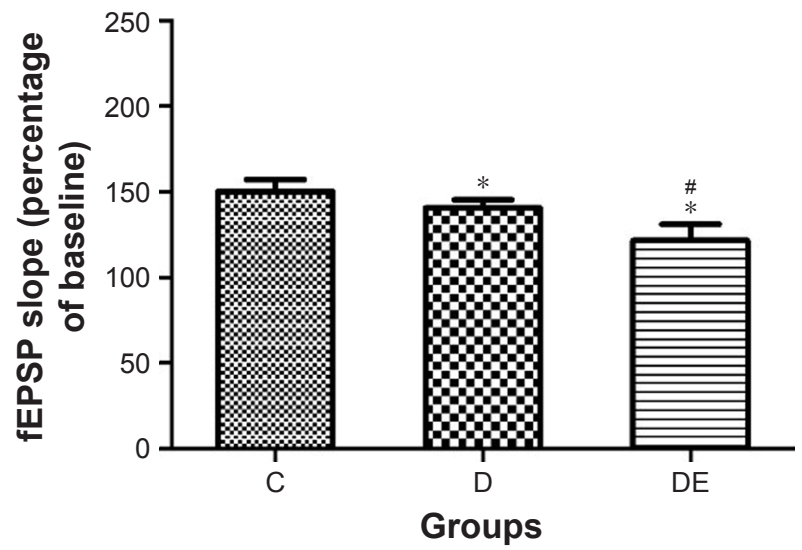

Figure 5 Effects of ECS on PTP.

Notes: Baseline fEPSPs were recorded for $30 \mathrm{~s}$, and poststimulation fEPSPs were recorded for $60 \mathrm{~s}$. (A-C) PTP was recorded in each group. Original traces of fEPSP were recorded, and baseline trace (trace I) and poststimulation trace (trace 2) were exhibited. Scale bar was set as $5 \mathrm{~ms}$ for the horizontal line and I $\mathrm{mV}$ for the vertical line. (D) Statistical analysis of PTP for different groups. Group C, control group with healthy rats; Group D, rats with depression-like behavior; Group DE, rats with depressionlike behavior undergoing ECS. $* P<0.05$ compared with group $C$; ${ }^{P}<0.05$ compared with group $D$.

Abbreviations: ECS, electroconvulsive shock; fEPSPs, field excitatory postsynaptic potentials; PTP, post-tetanic potentiation.

such as CUMS procedure, will induce less LTP impairment and LTD enhancement.

Except LTP and LTD, depotentiation is another important form of synaptic plasticity but has often been neglected. It has been proved that depotentiation and LTD contribute to subserving forgetting. ${ }^{8,23}$ However, what should be stressed is that depotentiation and LTD are two related but distinct forms of synaptic weakening due to different specific pathways for LTD and depotentiation. ${ }^{40,41}$ In our study, depotentiation was enhanced in group DE, indicating that LTP was not stable and could be easily reversed with ECS administration. As far as we know, this is the first time to confirm that depotentiation is responsible for learning and memory impairment induced by ECS. Combined with aforementioned findings, ECS-induced learning and memory impairment is mediated by metaplasticity and depotentiation. It is well received that either metaplasticity or depotentiation depends on NMDA receptors. ${ }^{42}$ What is more, previous research found the 
intracellular signaling pathway of NMDA receptor, such as mitogen-activated protein kinase (MAPK) involved in the LTP, LTD, and depotentiation, although different specific pathways for different types of synaptic plasticity (RasErk1/2 for LTP, Rap1-p38 for LTD, and Rap2-JNK for depotentiation).$^{41}$ It is reasonable to speculate that NMDAR and its downstream proteins, such as MAPK, play an important role in ECS-induced learning and memory. However, it still needs further study to clarify how ECS induces learning and memory impairment via regulating NMDA receptors' function and MAPK signaling.

LTP, LTD, and depotentiation are long-term synaptic plasticities. Accumulative evidence suggested that short-term synaptic plasticity also involved the process of learning and memory. ${ }^{43}$ Unlike long-term synaptic plasticity, short-term synaptic plasticity, such as PTP, mainly reflects presynaptic transmitter quanta release. ${ }^{17}$ In our previous studies, Luo et al ${ }^{18}$ found that ECS induced glutamate decrease in hippocampus. Furthermore, Zhu et $\mathrm{al}^{44}$ found that ECS downregulated the expression of GLT-1 (a type of glia glutamate transporters), which induced glutamate accumulation in the synaptic cleft. It is reasonable to speculate that transmitter release will decrease after ECS. In the present study, we found that PTP was impaired in group DE, which reflected a decrease in the probability of glutamate release of available quanta. These results confirmed our aforementioned assumption and demonstrated that learning and memory impairment induced by ECS is mediated partly by PTP impairment or glutamate release decrease.

It has been reported that anesthetics, such as propofol, thiopental could alleviate the ECS-induced learning and memory impairment. ${ }^{36,37}$ However, a previous study found that anesthetics might influence the antidepressant of ECS. ${ }^{38}$ How to alleviate ECS-induced learning and memory impairment without antidepressant efficacy compromise needs to be further investigated. Also, several limitations exit in this study. First, male rats were only included in this experiment due to the effects of estrogen on the learning and memory, so the results cannot extend to females. Second, the synaptic plasticity changes were explored to illuminate the underlying mechanism of ECS-induced learning and memory impairment; however, the time-related changes in ECS were not investigated in this study.

\section{Conclusion}

Our study demonstrates that ECS induces various synaptic plasticity changes, which involve both presynaptic and postsynaptic mechanisms. Postsynaptic LTP impairment, LTD and depotentiation enhancement, and presynaptic PTP impairment possibly contribute to learning and memory impairment induced by ECS.

\section{Acknowledgments}

The authors are most grateful to Jingyuan Chen, PhD, of the Department of Anesthesiology of The First Affiliated Hospital of Chongqing Medical University, Chonqing, China, for providing useful suggestions in this article. This work was supported by the National Natural Science Foundation of China grant (No 81271501), as well as a grant from National Key Clinical Specialty Construction Project (No 2011-170) and Chongqing Medical Key Discipline Construction Project (No 2007-2).

\section{Disclosure}

The authors report no conflicts of interest in this work.

\section{References}

1. Kessler RC, Berglund P, Demler O, et al. The epidemiology of major depressive disorder: results from the National Comorbidity Survey Replication (NCS-R). JAMA. 2003;289(23):3095-3105.

2. Thapar A, Collishaw S, Pine DS, Thapar AK. Depression in adolescence. Lancet. 2012;379(9820):1056-1067.

3. Cipriani A, Zhou X, Del GC, et al. Comparative efficacy and tolerability of antidepressants for major depressive disorder in children and adolescents: a network meta-analysis. Lancet. 2016;388(10047):881-890.

4. Sackeim HA, Prudic J, Fuller R, Keilp J, Lavori PW, Olfson M. The cognitive effects of electroconvulsive therapy in community settings. Neuropsychopharmacology. 2007;32(1):244-254.

5. Dudai Y. Molecular bases of long-term memories: a question of persistence. Curr Opin Neurobiol. 2002;12(2):211-216.

6. Bliss TV, Collingridge GL. A synaptic model of memory: long-term potentiation in the hippocampus. Nature. 1993;361(6407):31-39.

7. Andersen N, Krauth N, Nabavi S. Hebbian plasticity in vivo: relevance and induction. Curr Opin Neurobiol. 2017;45:188-192.

8. Huang CC, Hsu KS. Progress in understanding the factors regulating reversibility of long-term potentiation. Rev Neurosci. 2001;12(1): $51-68$.

9. Fujii S, Saito K, Miyakawa H, Ito K, Kato H. Reversal of long-term potentiation (depotentiation) induced by tetanus stimulation of the input to CA1 neurons of guinea pig hippocampal slices. Brain Res. 1991; 555(1):112-122.

10. Chen YL, Huang CC, Hsu KS. Time-dependent reversal of long-term potentiation by low-frequency stimulation at the hippocampal mossy fiber-CA3 synapses. J Neurosci. 2001;21(11):3705-3714.

11. Burette F, Jay TM, Laroche S. Reversal of LTP in the hippocampal afferent fiber system to the prefrontal cortex in vivo with low-frequency patterns of stimulation that do not produce LTD. J Neurophysiol. 1997; 78(2):1155-1160

12. Lin $\mathrm{CH}$, Lee CC, Gean PW. Involvement of a calcineurin cascade in amygdala depotentiation and quenching of fear memory. Mol Pharmacol. 2003;63(1):44-52.

13. Wagner JJ, Alger BE. Homosynaptic LTD and depotentiation: do they differ in name only. Hippocampus. 1996;6:24-29.

14. Malenka RC, Bear MF. LTP and LTD: an embarrassment of riches Neuron. 2004;44(1):5-21.

15. Li W, Liu L, Liu YY, et al. Effects of electroconvulsive stimulation on long-term potentiation and synaptophysin in the hippocampus of rats with depressive behavior. $J E C T$. 2012;28(2):111-117. 
16. Regehr WG. Short-term presynaptic plasticity. Cold Spring Harb Perspect Biol. 2012;4(7):a005702.

17. Zucker RS, Regehr WG. Short-term synaptic plasticity. Annu Rev Physiol. 2002;64:355-405.

18. Luo J, Min S, Wei K, Li P, Dong J, Liu YF. Propofol protects against impairment of learning-memory and imbalance of hippocampal Glu/ GABA induced by electroconvulsive shock in depressed rats. J Anesth. 2011;25(5):657-665.

19. Mao QQ, Xian YF, Ip SP, Tsai SH, Che CT. Long-term treatment with peony glycosides reverses chronic unpredictable mild stress-induced depressive-like behavior via increasing expression of neurotrophins in rat brain. Behav Brain Res. 2010;210(2):171-177.

20. Zhang F, Luo J, Min S, Ren L, Qin P. Propofol alleviates electroconvulsive shock-induced memory impairment by modulating proBDNF/ mBDNF ratio in depressive rats. Brain Res. 2016;1642:43-50.

21. Tsien JZ, Huerta PT, Tonegawa S. The essential role of hippocampal CA1 NMDA receptor-dependent synaptic plasticity in spatial memory. Cell. 1996;87(7):1327-1338.

22. Liu X, Sandkühler J. Characterization of long-term potentiation of C-fiber-evoked potentials in spinal dorsal horn of adult rat: essential role of NK1 and NK2 receptors. J Neurophysiol. 1997;78(4):1973-1982.

23. Cunha-Reis D, Aidil-Carvalho MF, Ribeiro JA. Endogenous inhibition of hippocampal LTD and depotentiation by vasoactive intestinal peptide VPAC1 receptors. Hippocampus. 2014;24(11):1353-1363.

24. Wang CC, Weyrer C, Paturu M, Fioravante D, Regehr WG. Calciumdependent protein kinase $\mathrm{C}$ is not required for post-tetanic potentiation at the hippocampal CA3 to CA1 synapse. J Neurosci. 2016;36: 6393-6402.

25. Dwivedi $Y$, Zhang H. Altered ERK1/2 signaling in the brain of learned helpless rats: relevance in vulnerability to developing stress-induced depression. Neural Plast. 2016;2016:7383724.

26. Riad M, Kobert A, Descarries L, Boye S, Rompré PP, Lacaille JC. Chronic fluoxetine rescues changes in plasma membrane density of 5-HT1A autoreceptors and serotonin transporters in the olfactory bulbectomy rodent model of depression. Neuroscience. 2017;356:78-88.

27. Yang $\mathrm{L}, \mathrm{Xu} \mathrm{T}$, Zhang $\mathrm{K}$, et al. The essential role of hippocampal alpha6 subunit-containing GABAA receptors in maternal separation stressinduced adolescent depressive behaviors. Behav Brain Res. 2016;313: $135-143$.

28. Wang JM, Yang LH, Zhang YY, et al. BDNF and COX-2 participate in anti-depressive mechanisms of catalpol in rats undergoing chronic unpredictable mild stress. Physiol Behav. 2015;151:360-368.

29. Kendler KS, Hettema JM, Butera F, Gardner CO, Prescott CA. Life event dimensions of loss, humiliation, entrapment, and danger in the prediction of onsets of major depression and generalized anxiety. Arch Gen Psychiatry. 2003;60(8):789-796.
30. Gao X, Zhuang FZ, Qin SJ, et al. Dexmedetomidine protects against learning and memory impairments caused by electroconvulsive shock in depressed rats: Involvement of the NMDA receptor subunit 2B (NR2B)ERK signaling pathway. Psychiatry Res. 2016;243:446-452.

31. Stewart C, Jeffery K, Reid I. LTP-like synaptic efficacy changes following electroconvulsive stimulation. Neuroreport. 1994;5(9):1041-1044.

32. Stewart C, Reid I. Electroconvulsive stimulation and synaptic plasticity in the rat. Brain Res. 1993;620(1):139-141.

33. Abraham WC, Bear MF. Metaplasticity: the plasticity of synaptic plasticity. Trends Neurosci. 1996;19(4):126-130.

34. Ren L, Zhang F, Min S, Hao X, Qin P, Zhu X. Propofol ameliorates electroconvulsive shock-induced learning and memory impairment by regulation of synaptic metaplasticity via autophosphorylation of CaMKIIa at Thr 305 in stressed rats. Psychiatry Res. 2016;240:123-130.

35. Xu L, Anwyl R, Rowan MJ. Behavioural stress facilitates the induction of long-term depression in the hippocampus. Nature. 1997; 387(6632):497-500.

36. Stripp TK, Jorgensen MB, Olsen NV. Anaesthesia for electroconvulsive therapy - new tricks for old drugs: a systematic review. Acta Neuropsychiatr. 2018;30(2):61-69.

37. Luo J, Min S, Wei K, et al. Propofol prevents electroconvulsive-shockinduced memory impairment through regulation of hippocampal synaptic plasticity in a rat model of depression. Neuropsychiatr Dis Treat. 2014;10:1847-1859.

38. Rasmussen KG. Propofol for ECT anesthesia a review of the literature. JECT. 2014;30(3):210-215.

39. Kim JJ, Diamond DM. The stressed hippocampus, synaptic plasticity and lost memories. Nat Rev Neurosci. 2002;3(6):453-462.

40. Zhu JJ, Qin Y, Zhao M, Van Aelst L, Malinow R. Ras and Rap control AMPA receptor trafficking during synaptic plasticity. Cell. 2002; 110(4):443-455.

41. Zhu Y, Pak D, Qin Y, et al. Rap2-JNK removes synaptic AMPA receptors during depotentiation. Neuron. 2005;46(6):905-916.

42. Philpot BD, Sekhar AK, Shouval HZ, Bear MF. Visual experience and deprivation bidirectionally modify the composition and function of NMDA receptors in visual cortex. Neuron. 2001;29(1):157-169.

43. Le BL, Léger L, Luppi PH, Fort P, Malleret G, Salin PA. Genetic deletion of melanin-concentrating hormone neurons impairs hippocampal short-term synaptic plasticity and hippocampal-dependent forms of short-term memory. Hippocampus. 2015;25(11):1361-1373.

44. Zhu X, Hao X, Luo J, Min S, Xie F, Zhang F. Propofol inhibits inflammatory cytokine-mediated glutamate uptake dysfunction to alleviate learning/memory impairment in depressed rats undergoing electroconvulsive shock. Brain Res. 2015;1595:101-109.
Neuropsychiatric Disease and Treatment

\section{Publish your work in this journal}

Neuropsychiatric Disease and Treatment is an international, peerreviewed journal of clinical therapeutics and pharmacology focusing on concise rapid reporting of clinical or pre-clinical studies on a range of neuropsychiatric and neurological disorders. This journal is indexed on PubMed Central, the 'PsycINFO' database and CAS,

\section{Dovepress}

and is the official journal of The International Neuropsychiatric Association (INA). The manuscript management system is completely online and includes a very quick and fair peer-review system, which is all easy to use. Visit http://www.dovepress.com/testimonials.php to read real quotes from published authors. 